\title{
Effectiveness of Biological Agents in the Treatment of Pediatric Patients with Crohn's Disease and Anal Fistulae
}

\author{
Nobuyasu Arai Takahiro Kudo Kazuhide Tokita Reiko Kyodo \\ Masamichi Sato Eri Miyata Kenji Hosoi Tamaki lkuse Keisuke Jimbo \\ Yoshikazu Ohtsuka Toshiaki Shimizu \\ Department of Pediatrics, Juntendo University Faculty of Medicine, Tokyo, Japan
}

\author{
Keywords \\ Crohn's disease · Anal fistula · Biological agents · Quality of \\ life
}

\begin{abstract}
Introduction: Anal fistulae have a significant impact on the quality of life of patients with Crohn's disease (CD). In this cross-sectional study, we aimed to determine whether biological agents were effective in treating anal fistulae in patients with CD. Methods: Fifty-three patients diagnosed with CD were retrospectively enrolled. Their data regarding symptoms, treatments, and disease progression from January 2007 to December 2016 were reviewed from the medical records. Fifteen (28\%) patients with CD were complicated by anal fistulae. Results: The male-to-female ratio was 13:2, and the mean age at onset was 11 years and 6 months. Among the 15 patients, 14 (93\%) had anal fistulae as an initial symptom. Almost all patients were treated by providing elemental diet, 5-aminosalicylic acid, and steroids as induction therapy. Biological agents were used in 8 patients (53.3\%), and fistula closure was confirmed in all of them. Among the 7 patients not treated with biological agents, 1 (14.3\%) had a recurrent anal fistula, while another had incomplete fistula closure. Regarding surgical management, 2 patients were treated using the
\end{abstract}

\begin{tabular}{ll}
\hline karger@karger.com & (c) 2021 The Author(s) \\
www.karger.com/dig & Published by S. Karger AG, Basel \\
& $\begin{array}{l}\text { This is an Open Access article licensed under the Creative Commons } \\
\text { Attribution-NonCommercial-4.0 International License (CC BY-NC) } \\
\text { (http://www.karger.com/Services/OpenAccessLicense), applicable to } \\
\text { the online version of the article only. Usage and distribution for com- } \\
\text { mercial purposes requires written permission. }\end{array}$
\end{tabular}

seton method, and no patients required a colostomy. Conclusion: Treatment with biological agents is highly effective concerning the closure of anal fistulae in patients with $C D$, and reducing pain may improve their quality of life.

(c) 2021 The Author(s)

Published by S. Karger AG, Basel

\section{Introduction}

An anal fistula greatly impacts a patient's quality of life due to behavioral restrictions, as a result of pain and secretions following a diagnosis of Crohn's disease (CD). The mortality rate of patients with CD lesions in the anal region was reported to be $15-50 \%$ overseas [1]. According to the report by the Japan Child Inflammatory Bowel Disease Registry Research [2], anal fistula complications are relatively common, occurring at a rate of $33.3 \%$ and often preceding other symptoms. There are several reports stating that biological agents are effective for induction or maintenance therapy in pediatric patients with active perianal fistula lesions and in those who had undergone surgical interventions [3]. In this study, we aimed to examine the effectiveness of biological agents for anal fistulae associated with CD. 


\section{Materials and Methods}

\section{Patients}

We conducted a retrospective study on pediatric patients with $\mathrm{CD}$ with anal fistulae observed in our department. Among 53 patients diagnosed with CD in our department between January 2007 and December 2016, the medical records of 15 patients (28\%) who had concurrent anal fistulae were reviewed. Adult cases of CD were excluded. Our study protocol complied with the ethical guidelines of the Declaration of Helsinki and was approved by the Ethics Committee of Juntendo University Hospital (approval number: 19-208). We disclosed the contents of this study and provided the opportunity to our participants to refuse or discontinue participation. The variables of interest were the following: time of initial symptoms; blood test results; endoscopic findings, such as lesion site; presence of a growth disorder; treatments used, such as 5-aminosalicylic acid (5-ASA), steroids, biological agents, and nutritional therapy; and details on the clinical course including anal fistula closure. We also summarized the following characteristics for each patient: background, reason for induction, timing of induction, and effectiveness of treatment. Overall, 8 out of 15 patients who had used biological agents were also retrospectively studied. In this study, growth disorder was defined as height $<-1.5$ SD.

\section{Diagnosis}

The diagnosis of CD was based on the following criteria [4]:

- Longitudinal ulceration. Mucosal cobblestoning. Noncaseating epithelioid cell granuloma.

- Irregular to widespread gastrointestinal tract round or aphthoid ulcer. Characteristic anal lesion. Characteristic gastroduodenal lesion. Definitive diagnosis: Presence of major criterion A or B. Presence of major criterion $\mathrm{C}$ and minor criterion a or b. Presence of all minor criteria.

\section{Statistical Analysis}

For the descriptive statistical analysis, average values and standard deviations were calculated using Microsoft ${ }^{\circledR}$ Excel 2010 (Microsoft Corp., Redmond, WA, USA).

\section{Results}

In total, the data of 53 patients with CD diagnosed at our department (male, 37; female, 16; age at onset, $10.5 \pm$ 4.1 years [range, $0-20$ years]) were assessed.

Table 1 shows the background characteristics of the 15 patients with anal fistulae (sex ratio [male:female participants], 13:2; age at onset, $11.6 \pm 3.0$ years [range, $2-15$ years]; age at diagnosis, $12.7 \pm 3.0$ years [range, $4-16$ years]). Among the 15 patients, 14 (93.3\%) had anal fistulae as the initial symptom. Surgical management using the seton method was performed in 2 patients. Initial induction therapy of 5-ASA was administered to all 15 patients (100\%); 13 (86.7\%) and $10(66.7 \%)$ patients underwent elemental diet and steroid therapies, respectively. The sites of lesions by the Paris classification [5] and the
Table 1. Clinical background of 15 patients with CD who had an anal fistula

\begin{tabular}{lc}
\hline Characteristic & $N=15$ \\
\hline Sex & 13 male, 2 female \\
Age at onset, years & $11.6 \pm 3.0$ \\
$\quad$ Range, years & $2-15$ \\
Age at diagnosis, years & $12.7 \pm 3.0$ \\
$\quad$ Range, years & $4-16$ \\
An anal fistula is the first symptom & $14(93.3 \%)$ \\
Surgical treatment (seton method) & $2(13.3 \%)$ \\
Induction therapy & $13(86.7 \%)$ \\
$\quad$ ED & $15(100 \%)$ \\
5-ASA & $10(66.7 \%)$ \\
PSL &
\end{tabular}

Data are presented as means \pm standard deviation or as numbers and percentages. CD, Crohn's disease; ED, elemental diet; 5-ASA, 5-aminosalicylic acid; PSL, prednisolone.

Table 2. Background characteristics of 8 patients treated with biological agents

\begin{tabular}{lc}
\hline Characteristic & $N=8$ \\
\hline Sex & \\
$\quad$ Male & 6 \\
$\quad$ Female & 2 \\
Age at diagnosis, years & $12.1 \pm 4.0$ \\
$\quad$ Range, years & $2-15$ \\
Duration from diagnosis to introduction, months & $47 \pm 50.3$ \\
$\quad$ Range, months & $3-162$ \\
Formulation used & \\
$\quad$ IFX & 7 \\
$\quad$ ADA & 1 \\
Used PSL at the time of introduction & $4(50 \%)$ \\
PCDAI at the time of introduction (score) & $16.6 \pm 6.6$ \\
$\quad$ Range (score) & $10-27.5$ \\
Surgical treatment (seton method) & $1(12.5 \%)$ \\
\hline
\end{tabular}

Data are presented as means \pm standard deviations or as numbers and percentages. PSL, prednisolone; IFX, infliximab; ADA, adalimumab; PCDAI, Pediatric Crohn's Disease Activity Index.

presence of a growth disorder in the 15 patients are presented in Figure 1. Four (26.6\%) and 6 (40\%) patients had lesions in L2 and L3, respectively. Colonic lesions were observed in all patients, and a growth disorder was found in 7 patients $(46.6 \%)$.

Table 2 shows the background characteristics of the 8 patients treated with biological agents. An indication for 
Fig. 1. Area of the lesion by the Paris classification [4] and the presence or absence of a growth disorder in 15 pediatric patients with $\mathrm{CD}$ and anal fistula. The sites of the lesion were L2 and L3 in 4 (26.6\%) and 6 patients (40\%), respectively. Growth disorder was observed in 7 patients $(46.6 \%)$. $\mathrm{CD}$, Crohn's disease.
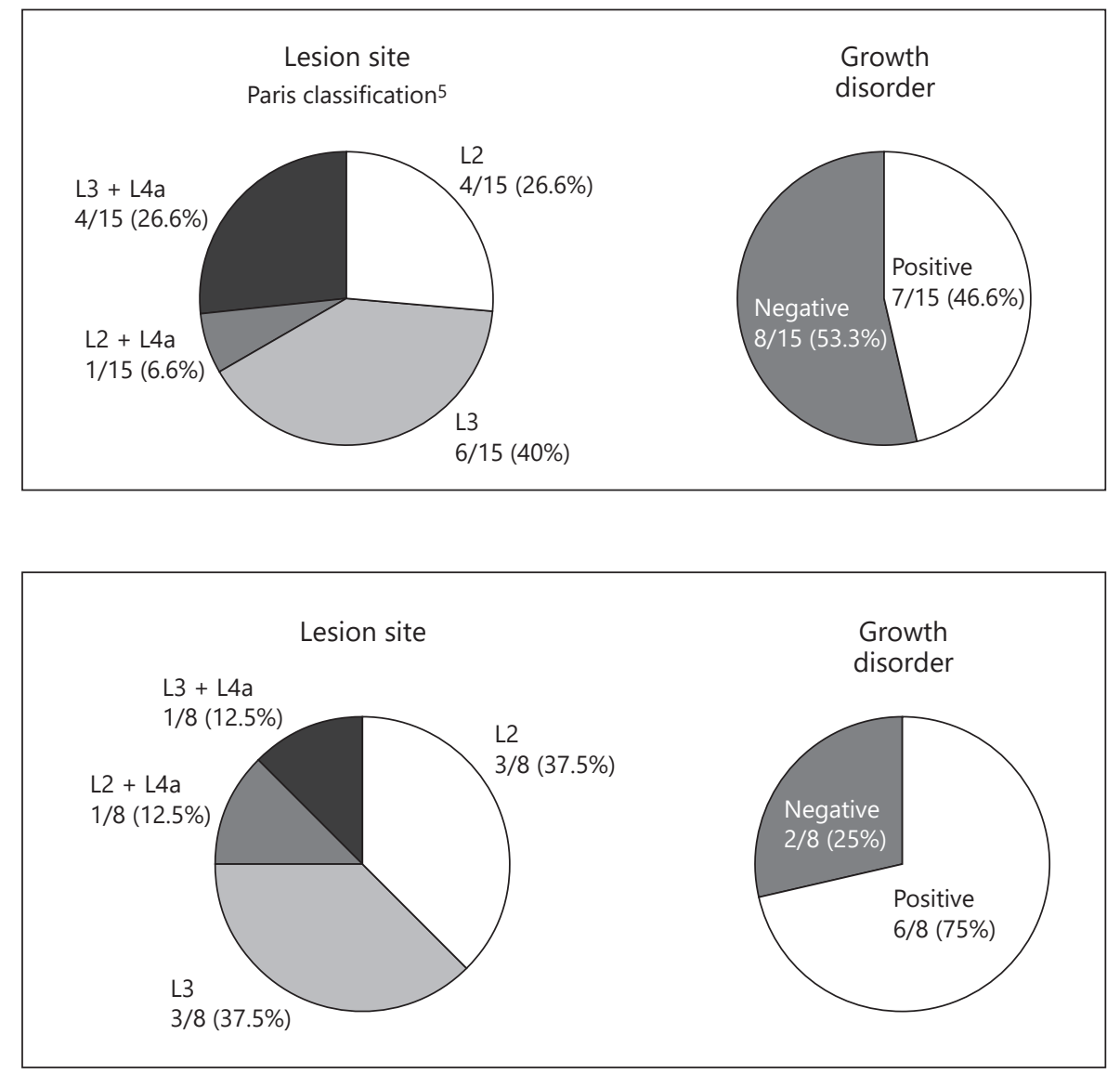

Fig. 2. Lesion sites in patients using biological agents and the presence or absence of a growth disorder. L2 and L3 were the most frequent sites, occurring in 3 patients each $(37.5 \%)$. A total of 6 patients $(75 \%)$ with growth disorders were admitted. the introduction of biological agents was relapsed or worsening symptoms in steroid-dependent patients, owing to the gradual reduction or abrupt discontinuation of steroids. In 7 of these patients, although their abdominal symptoms improved, the symptoms of the anal fistula remained unchanged and even worsened in some cases, which was attributed to the use of biological agents by these patients. The ratio of male-to-female participants treated with biological agents was 6:2, and the average age at diagnosis was $12.1 \pm 4.0$ years (range, $2-15$ years). The average time period from diagnosis to the introduction of biological agents was $47 \pm 50.3$ months (range, 3-162 months). A total of 7 patients received infliximab (IFX), while only 1 patient received adalimumab (ADA). In this study, there were 1 and 7 patients with simple and complex fistulas, respectively. Four patients (50\%) were receiving steroids at the time of biological agent introduction, and surgery using the seton method was performed in 1 patient. The Pediatric CD Activity Index (PCDAI) score was $16.6 \pm 6.6$ (range, 10-27.5) at the time of biological agent introduction, which corresponds to a mild severity in all patients. The site of the lesions in patients using biological agents and those with a growth disorder is shown in Figure 2. L2 and L3 were the most frequent lesion sites, and lesions at these sites occurred in 3 patients (37.5\%). Six patients $(75 \%)$ had a growth disorder.

Among the 7 patients who were not receiving biological agents, 1 patient (14.3\%) had an anal fistula that failed to close, while 6 patients (85.7\%) had confirmed closure of their fistulae. However, 1 patient had a recurrence of the fistula. Although the severity of the anal fistulae was not high in the 8 patients who had received biological agents, it was difficult to discontinue steroid administration in those whose fistulae did not improve and who had impaired quality of life as a result. However, all patients on biological agents eventually had their fistulae closed, and there was no recurrence thereafter. Particularly, at 8 weeks after the induction of IFX, 7 out of $8(87.5 \%)$ patients had their fistulae closed. Furthermore, all 4 patients who received steroids had their steroids discontinued, and all 6 patients with growth disorders had improved symptoms. 


\section{Discussion}

In our study, 14 patients (93.3\%) among the 15 diagnosed with CD complicated by an anal fistula in our department achieved fistula closure. Biological agents were introduced in 8 patients (53.3\%); all of them were able to withdraw from steroids. They all achieved fistula closure without any recurrence. Patients with CD complicated by anal fistula may have an early improvement of their anal fistula after biological agent introduction at an early stage, which shows a potential for a rapid quality of life advancement.

$\mathrm{CD}$ pathogenesis is associated with inflammatory cytokines, such as tumor necrosis factor-alpha (TNF- $\alpha$ ). TNF- $\alpha$ accumulates in the lamina propria of the inflamed mucosa in patients with CD [6]. Furthermore, the fecal levels of TNF- $\alpha$ increased in pediatric patients with CD [7]. TNF- $\alpha$ induces the expression of inflammatory chemokines and adhesion factors, which are involved in leukocyte migration to the sites of inflammation, leukocyte activation, and acute-phase protein production. AntiTNF- $\alpha$ monoclonal antibody, one of the biological agents used in cases of CD, has the following functions: (1) binding and neutralizing soluble TNF- $\alpha$ produced by activated macrophages, (2) a dissociating effect on TNF- $\alpha$ bound to the TNF- $\alpha$ receptor, and (3) an anti-inflammatory action due to the cytotoxic action on TNF- $\alpha$-producing cells [8].

Currently, anti-TNF- $\alpha$ monoclonal antibodies include IFX and ADA. They act as homologous biological agents and IFX is the most widely used. There are no studies comparing whether IFX or ADA should be used as induction therapy in biologically naïve patients with CD. According to the European Crohn's and Colitis Organization (ECCO) guidelines, biological agents are selected without definite criteria [9].

IFX is a chimeric anti-TNF- $\alpha$ monoclonal antibody, of which $75 \%$ is human derived and $25 \%$ mouse derived. In 1993, Derkx et al. were the first to report that IFX had a dramatic therapeutic effect in severe CD, which did not improve with steroid or immunomodulatory therapy [10]. Recently, induction and maintenance of remission was found to dramatically increase in adult patients with CD on IFX therapy [11]. In a study on patients with CD with an anal fistula, those in the IFX group (55\%) had a higher rate of fistula closure than those in the placebo group (13\%) [12].

In pediatric patients, there was a report [13] examining the efficacy of anti-TNF- $\alpha$ antibody preparations for those with CD with anal fistula. Especially, 60, 40, and $50 \%$ of patients with CD complicated with anal fistula were in remission, were keeping remission for 12 months and maintained remission afterward, and showed closure of the anal fistula, respectively. In our study, the anal fistula was closed in all cases using the anti-TNF- $\alpha$ antibody preparation, which was a better result than the corresponding in previous reports. Further, in 2007, Hyams et al. [14] reported the results of the REACH trial, evaluating the efficacy and safety of IFX in moderate-to-severe pediatric patients with $\mathrm{CD}$, confirming that IFX therapy was effective. Teitelbaum et al. [15] and Afzal et al. [16] reported that IFX was effective in the treatment of anal fistulae in 6 out of 8 patients (75\%). In addition, early introduction of IFX was effective for maintenance of longterm remission in 14 out of 15 patients [17]. A retrospective observational study at another institution [18] that examined the efficacy of IFX for patients with CD with anal fistula stated that the anal fistula was closed in $70 \%$ of patients and highlighted the efficacy of IFX in such patients. Similarly, in our study, closure of the anal fistula was confirmed in all 7 patients using IFX, and there was no recurrence. Therefore, IFX is considered to be effective for patients with $\mathrm{CD}$ with anal fistula without local infection. In particular, there have been many reports [15, $16,19]$ stating that IFX is effective in patients with anal fistulae, resulting in an improvement in their quality of life. Conversely, after making a thorough search, it was determined that the effectiveness of biological agents other than IFX for the treatment of anal fistulae remains unknown. A report by Ruemmele et al. [20] reported that $44.4 \%$ of patients with CD with anal fistula had their anal fistulae closed by 12 weeks after starting ADA, but the effects of using biologics other than IFX for anal fistula remain unknown. In our study, only 1 case used ADA, but closure was confirmed at 8 weeks after induction.

In Europe and the United States, the ECCO/ESPGHAN guidelines for children with CD were revised in 2014, and early administration of IFX had been recommended in adult and pediatric patients, in which resistance to treatment was expected [21]. In Japan, the guidelines for the treatment of pediatric CD [22] were revised in 2019. In principle, it was considered the use of biological agents when the performed treatment with nutritional therapy, 5-ASA preparations, steroids, and immunomodulators do not provide significant improvement. Among them, considering the use of biological agents for early introduction in patients with extensive small bowel lesions, steroid-resistant or dependent cases, and severe anal lesions is essential. However, consultation with a pediatric gastroenterologist is recommended before using biological agents.

Antibiotics may be useful for anal fistulae; however, no evidence has been provided regarding their use in pediat- 
ric patients. Treatment guidelines for pediatric $\mathrm{CD}$ with anal fistulae of mild severity, not affecting daily life, include incision, drainage, and antibiotic and surgical management using the seton method in moderate cases with persistent pain. In severe cases, not amenable to any of the various treatments, colectomy may be required.

In addition, a report by Forsdick et al. [23] showed a closure of anal fistula in $70 \%$ of patients with CD with anal fistula using IFX but recurrence in $1 / 4$ of those patients, which indicated the need for surgical treatment. Therefore, the treatment of $\mathrm{CD}$ with anal fistula required a combination of medical and surgical treatments. Our department has a policy of implementing the seton method in the case of refractory or recurrent anal fistula. However, we did not perform the seton procedure if the fistula improved after the induction of biological agents before the seton procedure.

In addition, it is recommended that immunosuppressive therapy with an immunomodulator or biological agent should be introduced after controlling local infections $[4,22]$. However, the timing and severity of symptoms at the introduction of immunosuppressive therapy have not been established.

Even in this study, IFX was used in 7 patients and was effective in all cases. Regarding the timing of IFX induction, it was reported that top-down therapy, which introduces IFX at an early stage of diagnosis, has a higher remission and mucosal clearance rate than step-up therapy, which introduces IFX after disease activity has increased and after starting steroids or immunomodulators [24].

However, IFX is a chimeric antibody containing 25\% amino acid sequences derived from mice, as aforementioned. Attention must be paid to allergic and delayed hypersensitivity reactions during its administration. In contrast, as ADA is a fully human antibody, serious reactions rarely occur [8].

In addition, there have been several reports stating that hepatosplenic T-cell lymphoma developed following combination treatment with anti-TNF- $\alpha$ antibody and immunomodulators $[25,26]$. Interestingly, combination therapy with immunomodulators could increase the risk of lymphoproliferative disorders compared to singleagent therapy with an anti-TNF- $\alpha$ antibody alone [27].

Based on these findings, careful examination of intractable cases is essential despite the effectiveness of biological agents in improving symptoms and achieving anal fistula closure. Therefore, it is important to screen patients for infectious diseases, such as viral hepatitis and tuberculosis, and include these conditions while obtaining informed consent.

Biologic Agents in Pediatric Crohn's Disease
The study had some limitations. As the targeted number of patients was small and the study was conducted at a single institution, regional characteristics and patient histories may have affected the results. In addition, the effectiveness of biological agents in anal fistulae has not been scientifically proven. In the future, it is necessary to validate these results by including a larger number of patients at multiple facilities beyond this region.

To conclude, after conducting a retrospective review of the data of 15 patients with CD complicated by anal fistulae in our department, we found that regardless of the severity of symptoms in patients, their quality of life could be rapidly improved by introducing biological agents at the early stages of CD. Based on the obtained results, we have changed our policy to introduce aggressive antiTNF- $\alpha$ agents in anal fistulas with improved perianal infection. Only a few pediatric patients with $C D$ received biological agents in this study. However, the optimal timing for the introduction of these agents remains unclear. This concept is an important issue and should be explored in future studies.

\section{Statement of Ethics}

This study protocol complied with the ethical guidelines of the Declaration of Helsinki and was approved by the Ethics Committee of Juntendo University Hospital (approval number: 19-208).

\section{Conflict of Interest Statement}

The authors have no conflicts of interest to declare.

\section{Funding Sources}

No funding was received for this study.

\section{Author Contributions}

Nobuyasu Arai, Takahiro Kudo, Kazuhide Tokita, Reiko Kyodo, Masamichi Sato, Eri Miyata, Kenji Hosoi, Tamaki Ikuse, and Keisuke Jimbo: substantial contributions to the conception or design of the work, or the acquisition, analysis, or interpretation of data for the work. Takahiro Kudo, Keisuke Jimbo, Yoshikazu Ohtsuka, and Toshiaki Shimizu: drafting the work or revising it critical for important intellectual content. All authors: final approval of the version to be published and agreement to be accountable for all aspects of the work in ensuring that questions related to the accuracy or integrity of any part of the work are appropriately investigated and resolved. 


\section{References}

1 Nielsen OH, Rogler G, Hahnloser D, Thomsen $O \varnothing$. Diagnosis and management of fistulizing Crohn's disease. Nat Clin Pract Gastroenterol Hepatol. 2009 Feb;6(2):92-106.

2 Arai K, Kunisaki R, Murakoshi T, Kakuta F, Hagiwara S, Shimizu T, et al. Japanese child inflammatory bowel disease registry research report 2015. J Pediatr Gastr Nutr. 2016;30(1):31.

3 Crandall W, Hyams J, Kugathasan S, Griffiths A, Zrubek J, Olson A, et al. Infliximab therapy in children with concurrent perianal Crohn disease: observations from REACH. J Pediatr Gastroenterol Nutr. 2009 Aug;49(2):183-90.

4 Ministry of Health, Labor and Welfare in Japan. Ulcerative colitis. Crohn's disease diagnostic criteria: treatment guidelines 2016. Japan. 2016.

5 Levine A, Griffiths A, Markowitz J, Wilson DC, Turner D, Russell RK, et al. Pediatric modification of the Montreal classification for inflammatory bowel disease: the Paris classification. Inflamm Bowel Dis. 2011 Jun; 17(6):1314-21.

6 Abraham C, Cho JH. Inflammatory bowel disease. N Engl J Med. 2009 Nov;361(21): 2066-78.

7 Braegger CP, Nicholls S, Murch SH, Stephens S, MacDonald TT. Tumour necrosis factor alpha in stool as a marker of intestinal inflammation. Lancet. 1992;339(8785):89-91.

8 Beppu T, Matsui T. Anti-tumor necrosis factor alpha antibody. J Pract Pharm. 2016;67(6): 2160-7.

9 Dignass A, Van Assche G, Lindsay JO, Lémann M, Söderholm J, F Colombel J, et al. The second European evidence-based consensus on the diagnosis and management of Crohn's disease: current management. J Crohns Colitis. 2010 Feb;4(1):28-62.

10 Derkx B, Taminiau J, Radema S, Stronkhorst A, Wortel C, Tytgat G, et al. Tumour-necrosis-factor antibody treatment in Crohn's disease. Lancet. 1993;342(8864):173-4.

11 Hanauer SB, Feagan BG, Lichtenstein GR, Mayer LF, Schreiber S, Colombel JF, et al.
Maintenance infliximab for Crohn's disease: the ACCENT I randomized trial. Lancet. 2002 May;359(9317):1541-9.

12 Present DH, Rutgeerts P, Targan S, Hanauer SB, Mayer L, Van Hogezand RA, et al. Infliximab for the treatment of fistulas in patients with Crohn's disease. N Engl J Med. 1999 May;340(18):1398-405.

13 Carnovale C, Maffioli A, Zaffaroni G, Mazhar F, Battini V, Mosini G, et al. Efficacy of tumour necrosis factor-alpha therapy in paediatric Crohn's disease patients with perianal lesions: a systematic review. Expert Opin Biol Ther. 2020 Mar;20(3):239-51.

14 Hyams J, Crandall W, Kugathasan S, Griffiths A, Olson A, Johanns J, et al. Induction and maintenance infliximab therapy for the treatment of moderate-to-severe Crohn's disease in children. Gastroenterology. 2007 Mar; 132(3):863-6.

15 Teitelbaum JE, Saeed S, Triantafyllopoulou $\mathrm{M}$, Daum F. Infliximab in pediatric Crohn disease patients with enterovesicular fistulas. J Pediatr Gastroenterol Nutr. 2007 Feb;44(2): 279-82.

16 Afzal NA, Shenoy MU, Haque S, Wilcox D, Shah N. Recognition and treatment of genitourinary complications in paediatric Crohn's disease using Infliximab. Acta Paediatr. 2010 Jul;99(7):1042-6.

17 Kugathasan S, Werlin SL, Martinez A, Rivera MT, Heikenen JB, Binion DG. Prolonged duration of response to infliximab in early but not late pediatric Crohn's disease. Am J Gastroenterol. 2000 Nov;95(11):3189-94.

18 Iwańczak BM, Ryżko J, Jankowski P, Sładek M, Wasilewska A, Szczepanik M, et al. Induction and maintenance infliximab therapy for the treatment of Crohn's disease with perianal fistulas in children: retrospective, multicenter study. Adv Clin Exp Med. 2016 May-Jun; 25(3):523-30

19 Kamm MA, Ng SC. Perianal fistulizing Crohn's disease: a call to action. Clin Gastroenterol Hepatol. 2008 Jan;6(1):7-10.
20 Ruemmele FM, Rosh J, Faubion WA, Dubinsky MC, Turner D, Lazar A, et al. Efficacy of adalimumab for treatment of perianal fistula in children with moderately to severely active Crohn's disease: results from IMAgINE 1 and IMAgINE 2. J Crohns Colitis. 2018 Nov 9; 12(10):1249-54.

21 Ruemmele FM, Veres G, Kolho KL, Griffiths A, Levine A, Escher JC, et al. Consensus guidelines of ECCO/ESPGHAN on the medical management of pediatric Crohn's disease. J Crohns Colitis. 2014 Oct;8(10):1179-207.

22 Arai K, Kudo T, Kumagai H, Saito H, Shimizu $\mathrm{H}$, Shimizu T, et al. Consensus report of the Japanese Society of pediatric inflammatory bowel disease on the management of pediatric Crohn's disease. Jpn J Pediatr Gastr Nutr. 2019 Dec;33(2):90-109.

23 Forsdick VK, Tan Tanny SP, King SK. Medical and surgical management of pediatric perianal crohn's disease: a systematic review. J Pediatr Surg. 2019 Dec;54(12):2554-8

24 D'Haens G, Baert F, van Assche G, Caenepeel $\mathrm{P}$, Vergauwe $\mathrm{P}$, Tuynman $\mathrm{H}$, et al. North-Holland Gut Club. Early combined immunosuppression or conventional management in patients with newly diagnosed Crohn's disease: an open randomized trial. Lancet. $2008 \mathrm{Feb}$ 371(9613):660-7.

25 Rosh JR, Gross T, Mamula P, Griffiths A, Hyams J. Hepatosplenic T cell lymphoma in adolescents and young adults with Crohn's disease: a cautionary tale? Inflamm Bowel Dis. 2007 Aug;13(8):1024-30.

26 Beaugerie L, Brousse N, Bouvier AM, Colombel JF, Lémann M, Cosnes J, et al. Lymphoproliferative disorders in patients receiving thiopurines for inflammatory bowel disease: a prospective observational cohort study. Lancet. 2009 Nov;374(9701):1617-25.

27 Parakkal D, Sifuentes H, Semer R, Ehrenpreis ED. Hepatosplenic T-cell lymphoma in patients receiving TNF- $\alpha$ inhibitor therapy: expanding the groups at risk. Eur J Gastroenterol Hepatol. 2011 Dec;23(12):1150-6. 